\title{
The Research Program at RIBRAS (Radioactive Ion Beams in Brasil)-III
}

Cite as: AIP Conference Proceedings 1224, 461 (2010); https://

doi.org/10.1063/1.3431452

Published Online: 03 May 2010

R. Lichtenthäler, A. Lépine-Szily, V. Guimarães, P. N. de Faria, D. R. Mendes, K. C. C. Pires, V. Morcelle, A. Barioni, M. C. Morais, R. Pampa Condori, M. Assunção, A. M. Moro, M. Rodríguez-Gallardo, and A. Arazi

ARTICLES YOU MAY BE INTERESTED IN

Comparison of the effects of couplings to breakup channels in reactions induced by ${ }^{6} \mathrm{Li}$ and ${ }^{6} \mathrm{He}$ on the same ${ }^{64} \mathrm{Zn}$ target

AIP Conference Proceedings 1681, 060006 (2015); https: / / doi.org/10.1063/1.4932292

Recent applications of four-body Continuum-Discretized Coupled-Channels calculations to ${ }^{6} \mathrm{He}$ reactions

AIP Conference Proceedings 1351, 29 (2011); https://

doi.org/10.1063/1.3608932

Elastic scattering of ${ }^{6} \mathrm{He}$ and ${ }^{7} \mathrm{Be}$ on a ${ }^{9} \mathrm{Be}$ target

AIP Conference Proceedings 1231, 173 (2010); https://

doi.org/10.1063/1.3428911 


\title{
The Research Program at RIBRAS (Radioactive Ion Beams in Brasil)-III
}

\author{
R. Lichtenthäler ${ }^{a}$, A. Lépine-Szily ${ }^{a}$, V. Guimarães ${ }^{a}$, P.N. de Faria ${ }^{a}$, \\ D.R.Mendes Jr. ${ }^{a}$, K.C.C. Pires ${ }^{a}$, V. Morcelle ${ }^{a}$, A. Barioni ${ }^{a}$, M.C. Morais ${ }^{a}$, \\ R.Pampa Condori, ${ }^{a}$ M. Assunção ${ }^{e}$, A.M. Moro ${ }^{b}$, M.Rodríguez-Gallardo ${ }^{b, c}$, \\ A. Arazi $^{d}$ \\ ${ }^{a}$ Instituto de Fisica da USP, São Paulo, Brazil, C.P. 66318, 05314-970 \\ ${ }^{b}$ Departamento de FAMN, Universidad de Sevilla, Apdo. 1065, E-41080, Sevilla, Spain \\ ${ }^{c}$ Instituto de Estructura de la Materia, CSIC, Serrano 123, E-28006 Madrid, Spain \\ ${ }^{d}$ Laboratorio TANDAR, Comision Nacional de Energia Atomica, Argentina \\ ${ }^{e}$ Departamento de Ciencias Exatas e da Terra, Unifesp, Campus de Diadema, São Paulo, Brazil
}

\begin{abstract}
A part of the research program developed in the RIBRAS facility over the last four years is presented. Experiments using radioactive secondary beams of light exotic nuclei such as ${ }^{6} \mathrm{He},{ }^{7} \mathrm{Be},{ }^{8} \mathrm{Li}$ on several targets have been performed. Elastic angular distributions have been analysed by the Optical Model and four body Continuous Discretized Coupled Channels Calculations (4b-CDCC) and the total reaction cross sections have been obtained. A comparison between the reaction cross sections of ${ }^{6} \mathrm{He}$ and other stable projectiles with medium-heavy targets was performed. Measurements of the proton transfer reaction ${ }^{12} \mathrm{C}\left({ }^{8} \mathrm{Li},{ }^{9} \mathrm{Be}\right){ }^{11} \mathrm{~B}$ are also presented.
\end{abstract}

Keywords: Enter Keywords here.

PACS: Replace this text with PACS numbers; choose from this list:

http://www.aip.org/pacs/index.html

\section{THE RIBRAS FACILITY}

The RIBRAS (Radioactive Ion Beams in Brasil) [1] facility consists of two superconducting solenoids capable of producing secondary beams of exotic nuclei. The solenoids are presently installed in one of the beam lines of the 8 MV Pelletron Tandem accelerator of the University of São Paulo. The solenoids make an in-flight selection of the reaction products emerging from the primary target and focus them on a secondary target where the reaction of interest takes place. The ${ }^{7} \mathrm{Li}$ primary beam and a ${ }^{9} \mathrm{Be}(\sim 12 \mu \mathrm{m})$ target are the standard to produce the ${ }^{6} \mathrm{He}$ and ${ }^{8} \mathrm{Li}$ beams via the one proton stripping ${ }^{9} \mathrm{Be}\left({ }^{7} \mathrm{Li},{ }^{6} \mathrm{He}\right)$ and one neutron pickup ${ }^{9} \mathrm{Be}\left({ }^{7} \mathrm{Li},{ }^{8} \mathrm{Li}\right)$ reactions. A ${ }^{3} \mathrm{He}$ gas primary target is used to produce the ${ }^{8} \mathrm{~B}$ and ${ }^{7} \mathrm{Be}$ beams by the ${ }^{3} \mathrm{He}\left({ }^{6} \mathrm{Li},{ }^{8} \mathrm{~B}\right)$ and ${ }^{3} \mathrm{He}\left({ }^{7} \mathrm{Li},{ }^{7} \mathrm{Be}\right)$ reactions. The primary beam traverses the primary target (see label (1) in Figure 1) and stops in the Faraday cup (3) which measures its current. The secondary

CP1224, International Symposium on Exotic Nuclei

edited by Yu. E. Penionzhkevich and S. M. Lukyanov

(C) 2010 American Institute of Physics 978-0-7354-0768-8/10/\$30.00 
particles are collected by the first solenoid within a cone between $2^{\circ} \leq \theta \leq 6^{\circ}$ defined by the Faraday cup and the collimator (2). The acceptance solid angle of the system is 30 msr. The first solenoid makes a magnetic-rigidity selection to focus the secondary beam in the secondary target position (7). Unwanted secondary particles are stopped in the blocker (5) and collimator (6). A second identical solenoid is mounted after the target (7) and will allow an additional filtering improving the purity of the secondary beams. Presently we operate only with the first solenoid.

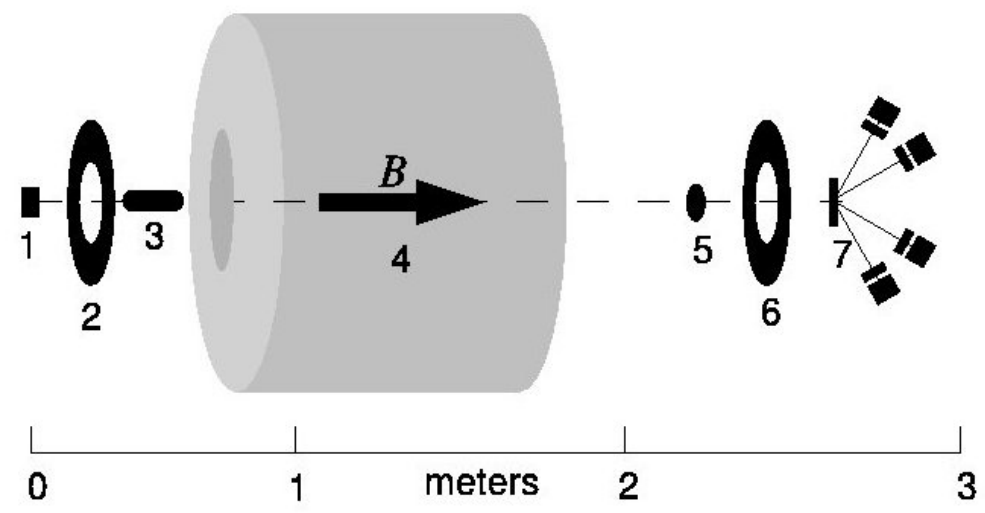

FIGURE 1. Scheme of the first solenoid RIBRAS system. -1-primary target, 2-collimator, 3-Farady cup, 4-solenoid, 5-unwanted-beam blocker, 6-collimator, 7-secondary target and detectors.

This system allows the production of beams of the light nuclei mentioned above with intensities of $10^{4}$ to $10^{6}$ pps and energies in the range 1-5 MeV/A.

\section{THE RESEARCH PROGRAM AT RIBRAS}

\section{Elastic scattering and Total Reaction Cross Section.}

Most of the experiments performed at RIBRAS consist of elastic scattering and nucleon transfer reactions measurements using light exotic beams such as ${ }^{6} \mathrm{He},{ }^{8} \mathrm{Li}$ and ${ }^{7} \mathrm{Be}$. The elastic scattering allows the study of the interacting potential and the total 
reaction cross section. We have been developing this program using the mediumheavy target ${ }^{120} \mathrm{Sn}$ [2], medium mass targets such as ${ }^{58} \mathrm{Ni}$ and ${ }^{51} \mathrm{~V}$ [3] and lighter targets such as ${ }^{27} \mathrm{Al}[4],{ }^{12} \mathrm{C}[5,6]$ and ${ }^{9} \mathrm{Be}[7]$.

The angular distributions have been analyzed by optical model and four-body CDCC calculations. In Figure 2 we present the elastic angular distributions for the ${ }^{6} \mathrm{He}+{ }^{120} \mathrm{Sn}$ system compared to Optical Model and 4b-CDCC calculations[8].
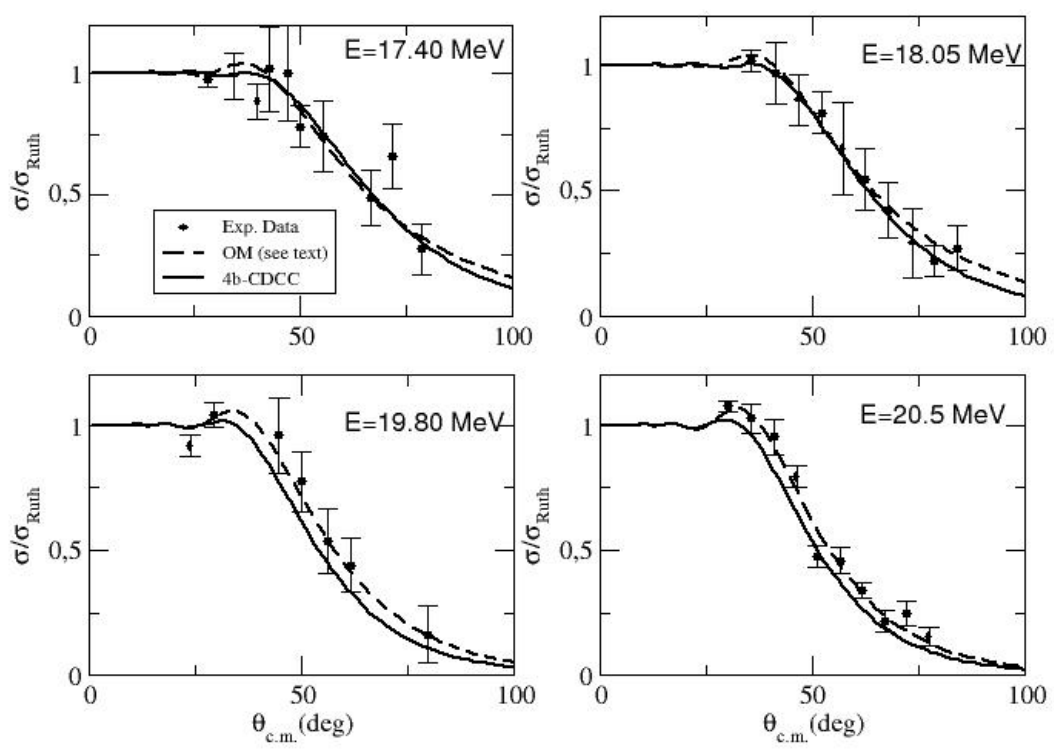

FIGURE 2. Elastic scattering angular distributions for the ${ }^{6} \mathrm{He}^{+120} \mathrm{Sn}$. Dashed line is Optical Model and solid line is the 4b-CDCC calculation [8].

To compare the reaction cross sections of different systems at different energies, it is convenient to rescale the cross sections and energies in order to overcome trivial effects due to the different sizes and energies with respect to the Coulomb barrier. We apply two methods to reduce the cross sections and energies $[9,10]$ : the first is to calculate the ratio to the square of the system radius $\sigma_{\text {red }}=\sigma /\left(A_{p}^{1 / 3}+A_{t}^{1 / 3}\right)^{2}$ and $E_{\text {red }}=E_{c m}\left(A_{p}^{1 / 3}+A_{t}^{1 / 3}\right) / Z_{p} Z_{t}$, the second is based on the Wong's [11] formula which depends on the quantity $\chi=\left(E-E_{C B}\right) / \eta w$ where $\eta w$ is the curvature of the barrier.

With both methods (see Figure 3) we observe an increase of the total reaction cross section for the system ${ }^{6} \mathrm{He}+{ }^{120} \mathrm{Sn}$ in comparison with the weakly-bound stable projectiles ${ }^{6,7} \mathrm{Li},{ }^{9} \mathrm{Be}$ and tightly bound projectiles as ${ }^{16} \mathrm{O}$ and alpha particles. A similar 
behavior was observed also for medium mass systems [9] and it was initially attributed to the larger breakup probability of ${ }^{6} \mathrm{He}$. However, there are strong indications that the neutron transfer stripping reactions to bound states are very important reaction channels contributing to the total reaction cross section of ${ }^{6} \mathrm{He}+\mathrm{X}$. A large yield of alpha particles was observed in the ${ }^{6} \mathrm{He}+{ }^{120} \mathrm{Sn}$ collision, with energies matching the ones expected from $2 n$-stripping reaction kinematics[2].

Reduced total reaction cross sections for medium-heavy mass systems
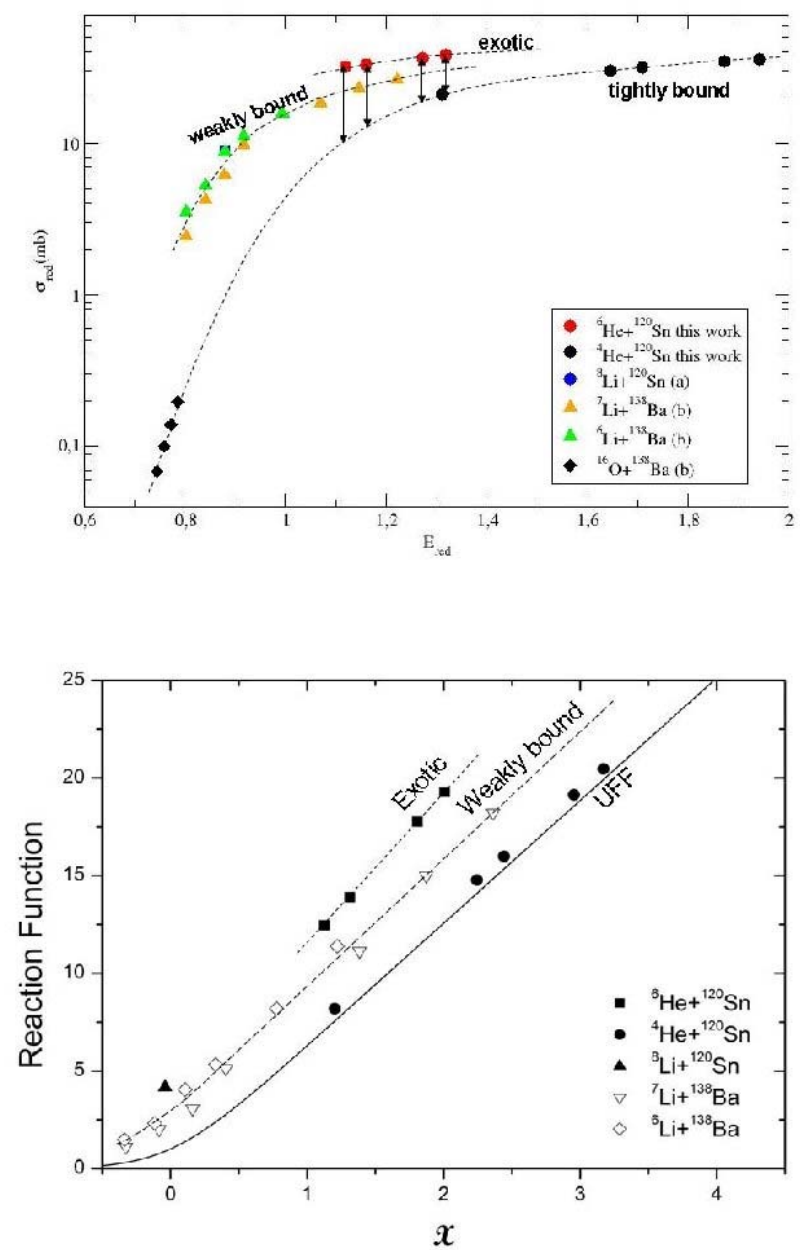

FIGURE 3. Total reduced reaction cross sections for medium-heavy mass systems with exotic, weakly bound and tightly bound projectiles. Dashed lines are guides to the eyes. Two reduction methods are used in the top and bottom figures (see text for more details). In the top figure, the arrows display the difference of the cross section between the exotic projectile ${ }^{6} \mathrm{He}$ and the tightly bound ${ }^{4} \mathrm{He}$. In the bottom figure, the solid line is the Universal Fusion Function (UFF) derived from the Wong formula [see Ref. 9]. (a) stands for Ref. [15] and (b) for Ref.[9] 
For lighter systems such as ${ }^{6} \mathrm{He}+{ }^{27} \mathrm{Al},{ }^{6} \mathrm{He}+{ }^{12} \mathrm{C}$ and ${ }^{6} \mathrm{He}+{ }^{9} \mathrm{Be}$ the situation is still inconclusive. There are some indications that the enhancement observed in ${ }^{6} \mathrm{He}+$ heavy targets could be smaller for lighter systems such as the ${ }^{6} \mathrm{He}+{ }^{27} \mathrm{Al}[4]$ and ${ }^{8} \mathrm{~B}+{ }^{12} \mathrm{C}$ [6], although a larger reduced reaction cross section has been obtained in some analysis [9].

\section{Nucleon Transfer Reactions}

Nucleon transfer reactions induced by exotic projectiles provide, in many cases, important spectroscopic information about states of nuclei not accessible by other means. In particular, the unstable lithium isotopes such as ${ }^{8,9} \mathrm{Li}[6,12,13,14]$ are important under the nuclear structure point of view and also for astrophysics as they could help to bridge the $\mathrm{A}=8$ gap. In Figure 4 we present an angular distribution of the proton transfer ${ }^{12} \mathrm{C}\left({ }^{8} \mathrm{Li},{ }^{9} \mathrm{Be}\right){ }^{11} \mathrm{~B}$ reaction measured at RIBRAS. As the vertex $\left\langle\left.{ }^{11} B\right|^{12} C>\right.$ is known, this reaction provides information of the $<\left.{ }^{8} \mathrm{Li}\right|^{9} \mathrm{Be}>$ spectroscopic factor, which normalizes the radiative capture ${ }^{8} \mathrm{Li}+p \rightarrow{ }^{9} \mathrm{Be}$ cross section. In Figure 5 we present the reaction rates for the above capture reaction derived using the spectroscopic factor $C^{2} S\left({ }^{9} \mathrm{Be}\right)=1.10(25)$ obtained from this work. We compare our results with other measurements.

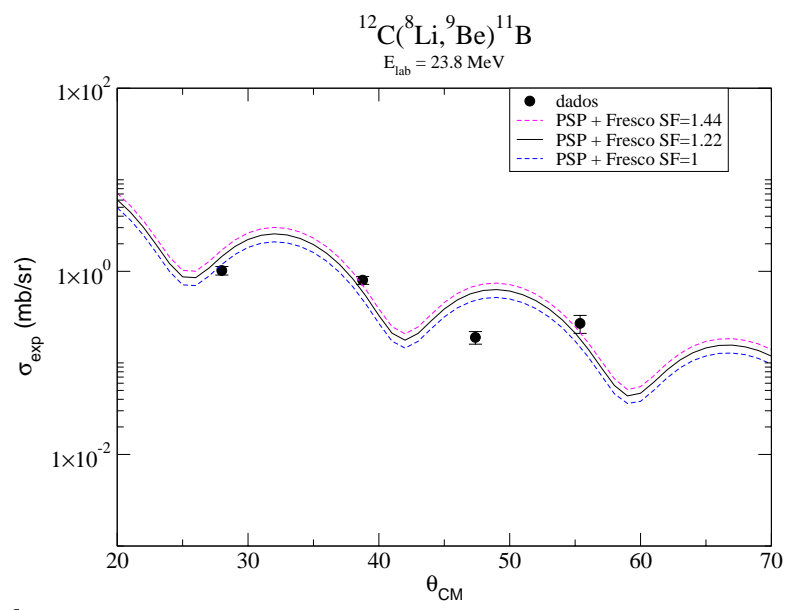

]

DWBA calculations. 


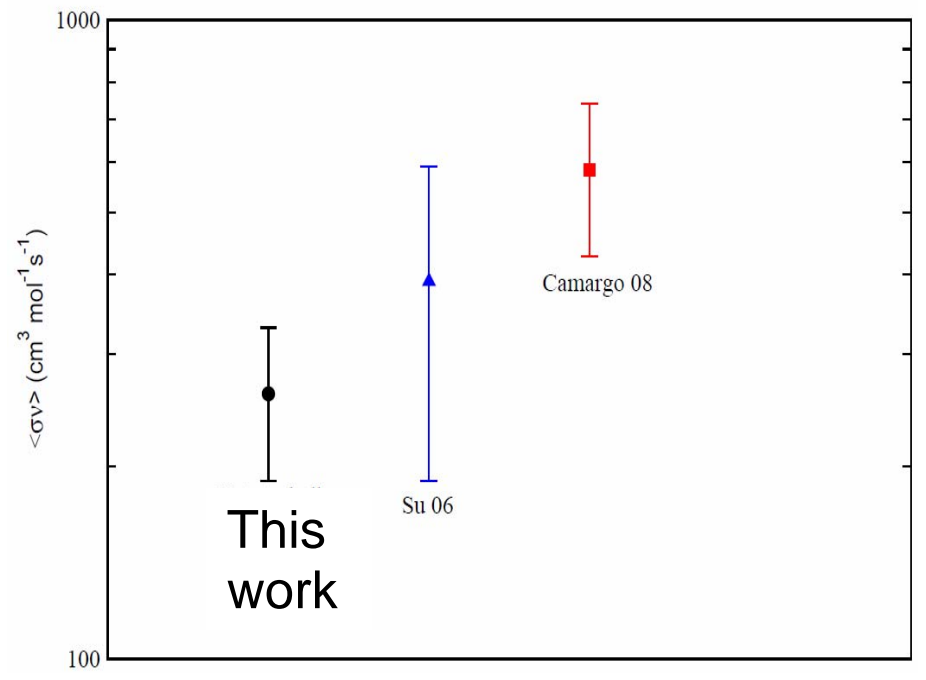

FIGURE 5. ${ }^{8} \mathrm{Li}+p \rightarrow{ }^{9} \mathrm{Be}$ reaction rate calculated at $\mathrm{T}_{9}=1$. Su06 stands for Ref. [13] and Camargo 08 for Ref. [14] .

\section{ACKNOWLEDGMENTS}

The authors thank the Fundação de Amparo à Pesquisa do Estado de São Paulo (FAPESP) and the Conselho Nacional de Desenvolvimento Científico e Tecnológico (CNPq) for financial support.

\section{REFERENCES}

1. R. Lichtenthäler et al., Eur. Phys. J. A 25,s01,733 (2005) and Nucl. Phys. News 15, 25 (2005).

2. R. Lichtenthäler et al., AIP Conference proceedings 1139, 76 (2009).

http://scitation.aip.org/dbt/dbt.jsp?KEY=APCPCS\&Volume=1139\&Issue=1

3. V. Morcelle, Master Thesis, Instituto de Física da USP (2007)

4. E. Benjamin et al., Physics Letters B647,30,(2007)

5. A. Barioni, PhD Thesis, Instituto de Física da USP (2009)

6. A. Barioni et al. Phys. Rev. C 80, 034617(2009)

7. K.C.C. Pires, $\mathrm{PhD}$ Thesis, in preparation

8. M. Rodríguez-Gallardo et al, Phys.Rev.C77, 064609(2008)

9. J.M.B. Shorto et al. Phys. Lett. B678(2009)77

10. P.R.S. Gomes et. al. Phys. Lett. B601, 20(2004)

11. C.Y. Wong, Phys. Rev. Lett. 31(1973)766

12. V. Guimarães et. al. Phys. Rev. C75, 054602(2007)

13. Su et al., Chin. Phys. Lett. 23,55(2006)

14. O. Camargo et. al. Phys. Rev. C78, 034605(2008)

15. P. Faria, PhD Thesis, Instituto de Física da USP (2008) 